\title{
Misdiagnosis of bradyarrhythmias in clinical practice in Iraq
}

\begin{abstract}
Background and objective: Bradyarrhythmias are one of the many causes of syncope, pre-syncope, and dizzy spells. Missing the diagnosis of bradyarrhythmias, an underlying etiology of those symptoms, may lead to serious complications and even mortality. This study aimed to set a standard case definition of bradyarrhythmias, which will improve patient survival.
\end{abstract}

Methods: Patients presented with partial or total loss of consciousness, who were misdiagnosed as other non-arrhythmic etiologies and later diagnosed as bradyarrhythmia, were included in this study. Diagnosis of bradyarrhythmias was reached by either 12 leads electrocardiography, Holter monitor, or electrophysiological study.

Results: A total of 150 patients who fulfilled the definition of missed diagnosis of bradyarrhythmias were included. A total of 100 males and 50 females were collected over 10 years. The pre arrhythmic over-diagnosis included transient ischemic attacks in 35 patients, vertebrobasilar insufficiency in 40 patients, vertigo in 30 patients, and other nonspecific diagnoses in 45 patients. The final diagnosis of bradyarrhythmias was reached by electrocardiogram only in 45 , Holter recording in 75 , and electrophysiological study in 30. All the diagnosed cases of bradyarrhythmias received permanent pacemaker therapy.

Conclusion: Misdiagnosis of bradyarrhythmias in patients presenting with syncope is not uncommon in clinical practice in Iraq, which may have a bad impact on patients' morbidity and mortality. Understanding the causative factors for the missed diagnosis can help set up an educational program to minimize this problem.

Keywords: Misdiagnosis; Bradyarrhythmia; Syncope; Dizzy spells.

\section{Introduction}

Bradyarrhythmias are of a wide range of electrocardiographic features, mechanisms, and clinical presentations. ${ }^{1-6}$ Syncope, pre syncope, and dizzy spells are the most common presenting symptoms. ${ }^{7-17}$ Due to the complexity and multiple etiologies of these symptoms, bradyarrhythmias are commonly misdiagnosed in clinical practice. ${ }^{2-5}$ Considering the bradyarrhythmia in the process of differentiating the causes of unexplained syncope, dizzy spells, and pre syncope can minimize this misdiagnosis and improves the prognosis and morbidities of bradyarrhythmias. ${ }^{8-17}$ Syncope is a syndrome with a relatively quiet sudden onset and of self -terminating period of a total loss of consciousness (TLOC) due to total cerebral hypoperfusion. $^{8-18}$ Other causes of TLOC should be considered in the differentiation of unexplained syncope. It is also important to remember that other conditions like epilepsy, metabolic disturbances, and psychological causes may cause TLOC. ${ }^{7-18}$ Syncope is not a diagnosis but is a feature of many underlying causes. ${ }^{8,9}$ Bradyarrhythmias are one of the underlying etiologies, and reaching the diagnosis of it as an etiology of syncope, pre syncope, and dizzy spells is highly important regarding further management and improving patient survival. On the

${ }^{1}$ Sulaimanya Heart Hospital and Alhassani Heart Center, Sulaimanya, I raq

* Correspondence: amaralhamdi@hotmail.com 
other hand, missing the diagnosis of bradyarrhythmias as an etiology of TLOC may well lead to serious morbidity and even an increased mortality rate. ${ }^{10-20}$ History taking, eye witness description, and basic cardiac investigations can lead to the diagnosis of bradyarrhythmias as a cause of syncope in around 30-40\% of syncope presented to the general physicians, emergency department, and general cardiologist. Still, a significant number of patients with bradyarrhythmias are missed during the first, second, and even third medical contacts. ${ }^{16,17,19,20}$ In this clinically based observational study, we reviewed 150 cases of misdiagnosed bradyarrhythmias causing TLOC, dizzy spells, or pre syncope and then diagnosed either by ECG, Holter monitor, or electrophysiological study (EPS). This study aimed to set up a clear definition of bradyarrhythmias to help to plan appropriate therapy.

\section{Methods}

This is a case series study where patients presented with misdiagnosed bradyarrhythmias were included. Patients were collected over a period from 19992019. The age of the patients ranged from 18-82 years, with a mean age of 45 years. Misdiagnosis of bradyarrhythmias is defined as patients presented with syncope, pre syncope, or dizzy spells and misdiagnosed as other non - arrhythmic etiologies and may be treated as such and later diagnosed as bradyarrhythmias causing these symptomatologies. The diagnosis of bradyarrhythmias is reached either with 12 leads ECG (ECG (Fukuda Denshi, Hongo, Bunkyo-ku, Tokyo, Japan), Holter monitor (TLC 5000, Contec Medical Systems, Qinhuangdao, China.) or electrophysiological study (CardioLab v5,2, GE Health care USA). The number of medical contacts each missed patient had before reaching the diagnosis of bradyarrhythmias was considered as first, second, or third medical contacts as the number of the patient visits to the medical practitioners who are either general practitioners (GP), general physician, clinical cardiologist or even coronary interventionist. The underlying etiologies which led to the misdiagnosis of bradyarrhythmias were assessed by considering the following possibilities; incomplete history taking including poor or lacking eye witness description, improper and non-careful 12 leads ECG inspection, nonefficient Holter recording and analysis, and non-realizing or considering the value of EPS in the diagnosis of bradyarrhythmias. Those patients with symptomatic bradyarrhythmias are treated with implantation of a permanent pacemaker according to the device guidelines of the ACC/AHA/HRS. ${ }^{1}$ The permanent pacemaker implantation was done under $2 \%$ Xylocaine local anesthetic and conscious sedation. Left subclavian vein picture was done, and two or single guide wires were introduced, and then either a single right ventricular (RV) lead was passed through peel away sheaths down to the RV apex (RVA), RV outflow (RVOT), or in few cases to the His bundle site. Pacemaker leads were active fixation type in the majority, and in a few, a passive lead was used. A direct cephalic vein cut down was done in some patients, and a single or two leads were introduced directly without peel away sheaths. The pacing and sensing parameters were measured in the single ventricular chamber $(\mathrm{V})$ or at the dual atrial $(\mathrm{A})$ and $\checkmark$ chambers. Value of pacing threshold of $1 \mathrm{v}$ or below is accepted, and a sense $A$ (at the RA) or $V$ (at the RV) value of $3 \mathrm{mv}$ and $6 \mathrm{mv}$ accordingly were accepted. The leads were fixed near its proximal end to the surrounding tissue by silk sutures and then connected to the pacemaker box which is implanted deep below the pectoral muscles. The subcutaneous tissue and the skin were sutured routinely. Patients discharged home the next day after pacemaker check and doing CXR to be seen in pacemaker follow up clinic after 12 days of implantation date, two months 
later, and then every six months. Symptoms reviewed and cardiac device interrogated to assess the percent of pacing and sensing in each chamber and routine check of other pacemaker and leads parameters. Patients were followed up for 1-10 years at the pacemaker follow up clinic at periods of 4-6 months where the symptoms were reviewed, the ECG was done, and the pacemaker was interrogated and programmed according to the patient's clinical condition. The ethical approval was obtained from the ethics committee of the Alhassani cardiac center. Regarding statistical analysis, a scientific calculator was used to calculate the percentages.

\section{Results}

A total of 150 patients (100 males and 50 females) with misdiagnosed bradyarrhythmias were collected over 10years by the author at the teaching hospitals of Al-Nahrain College of Medicine at Baghdad, Nasiriya heart center,
Nasiriya, Sulaimanya heart hospital and the private clinic of the author in Sulaimanya. The provisional diagnosis before the diagnosis of the bradyarrhythmias as a presumed cause of the patients' symptoms included transient ischemic attack (TIA) in 35 patient (23\%), vertigo in 30 patient (20\%), vertebrobasilar insufficiency in 40 patients (27\%) and other nonspecific diagnoses in 45 patients (30\%), as shown in Table 1 . Forty five patients were diagnosed as bradyarrhythmias by 12 leads ECG only, 75 patients by Holter monitor, and 30 patients by EPS. The diagnosis of bradyarrhythmias was missed in 40 patients during the first medical contact, in 100 patients during the second medical contact, and in 10 patients during a third medical contact. The underlying etiology of missing the diagnosis of these bradyarrhythmias is rather difficult to spot clearly. The trial to analyze the possible causes in each patient is shown in Table 2.

Table 1: Provisional diagnoses before the diagnosis of bradyarrhythmias was made.

\begin{tabular}{lcc}
\hline Provisional diagnosis & No. & (\%) $\mathbf{n}=\mathbf{1 5 0}$ \\
\hline TIA & 35 & 23.3 \\
Vertigo & 30 & 20.0 \\
Vertebral basilar insufficiency & 40 & 26.66 \\
Others nonspecific diagnoses & 45 & 30.0 \\
\hline
\end{tabular}

TIA, transient ischemic attacks

Table 2: The underlying etiologies of missed diagnosis of bradyarrhythmias.

\begin{tabular}{lll}
\hline Etiology & No. & (\%) \\
\hline Non - detailed history & 50 & 33.3 \\
Poor description of symptoms & 50 & 33.3 \\
Poor eye witness description & 50 & 33.3 \\
Non - careful ECG inspection & 50 & 33.3 \\
Non - efficient Holter analysis & 35 & 23.3 \\
Non - considering EPS & 15 & 10.0 \\
\hline
\end{tabular}

EPS: Electrophysiological study. ECG: electrocardiogram Multiple aetiologies are encountered in the same patient 
Multiple etiologies are encountered in some patients. Fifty patients (33\%) showed non detailed history taking and poor description of the symptoms and no or poor eye witness description in another 50 patients (33\%). Non-careful or quite quick inspection and interpretation of the series of the 12 leads ECGs was the reason in 50 patients (33\%). Non-efficient Holter data analysis was the cause in 35 patients (23\%), and not knowing or non-considering EPS as a diagnostic tool was responsible in 15 patients (10\%). In 65\%, multiple etiologies of the above mentioned were reported. All the 150 patients diagnosed as cases of bradyarrhythmias were treated by permanent pacemaker implantation. In 105, a left subclavian vein puncture was used to approach the venous access, and in 45, a direct cephalic vein cut-down was used. The modalities (types) of pacemaker implanted for each diagnosis are shown in Table 3. In 70 patients, a single chamber pacemaker of VVI mode was implanted, and in the other 80 patients, a dual chamber device of DDD mode according to the basic indication of the pacemaker following the device guidelines and the availability of the pacemaker type. In patients with atrial fibrillation and AV block, a single chamber device was used in 40 of the 70 patients with $\mathrm{VVI}$ mode. The remaining 30 patients of the $\mathrm{VVI}$ mode were either symptomatic bifascicular block in 10 , hypersensitive carotid body in 10 , or high grades AVB in 10 . The 80 patients who received a DDD pacemaker included 30 patients with sick sinus syndrome, 40 patients with high grades AVB, and 10 patients with symptomatic bifascicular block. Figures 1-4 are some examples of misdiagnosed patients with bradyarrhythmia confirmed by electrocardiograms, Holter monitoring or EP studies.

Table 3: The treatment of bradyarrhythmias by permanent pacemaker implantation.

VVI

70

40 A Fib with $\mathrm{CBH}$

10 Symptomatic bifascicular block

10 Carotid body hypersensitivity

10 High grades AVB

DDD

30 SSS

40 High grades AVB

10 Symptomatic bifascicular block

VVI: single ventricular chamber pacemaker code. DDD: dual chamber pacemaker code. A Fib: atrial fibrillation. $\mathrm{CHB}$, complete heart block. AVB: atrio-ventricular block. SSS: sick sinus syndrome ${ }^{21}$. 


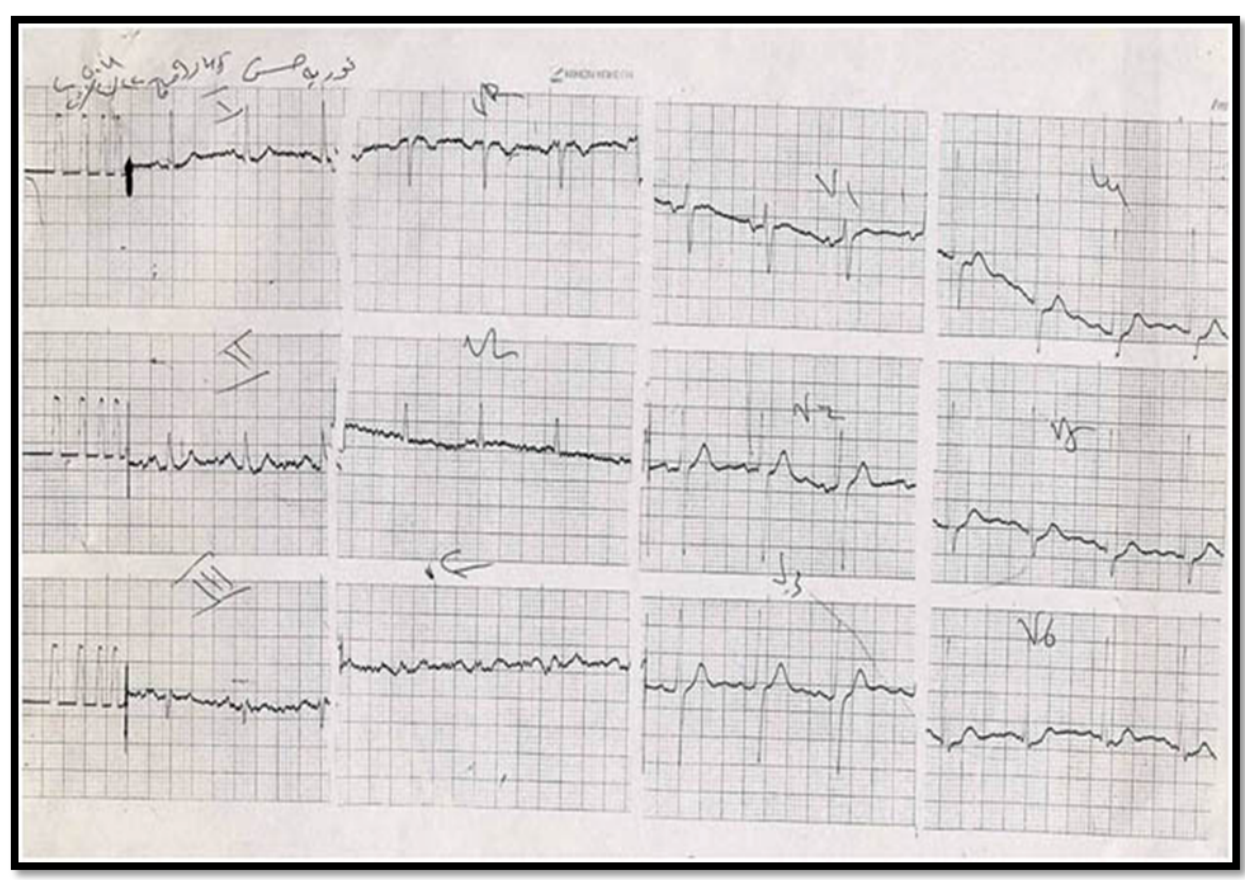

Figure 1 A: twelve leads ECG of 55 y old lady presented with pre syncope diagnosed as vertigo. Missed past history: old pure posterior MI indicated by R in V1.

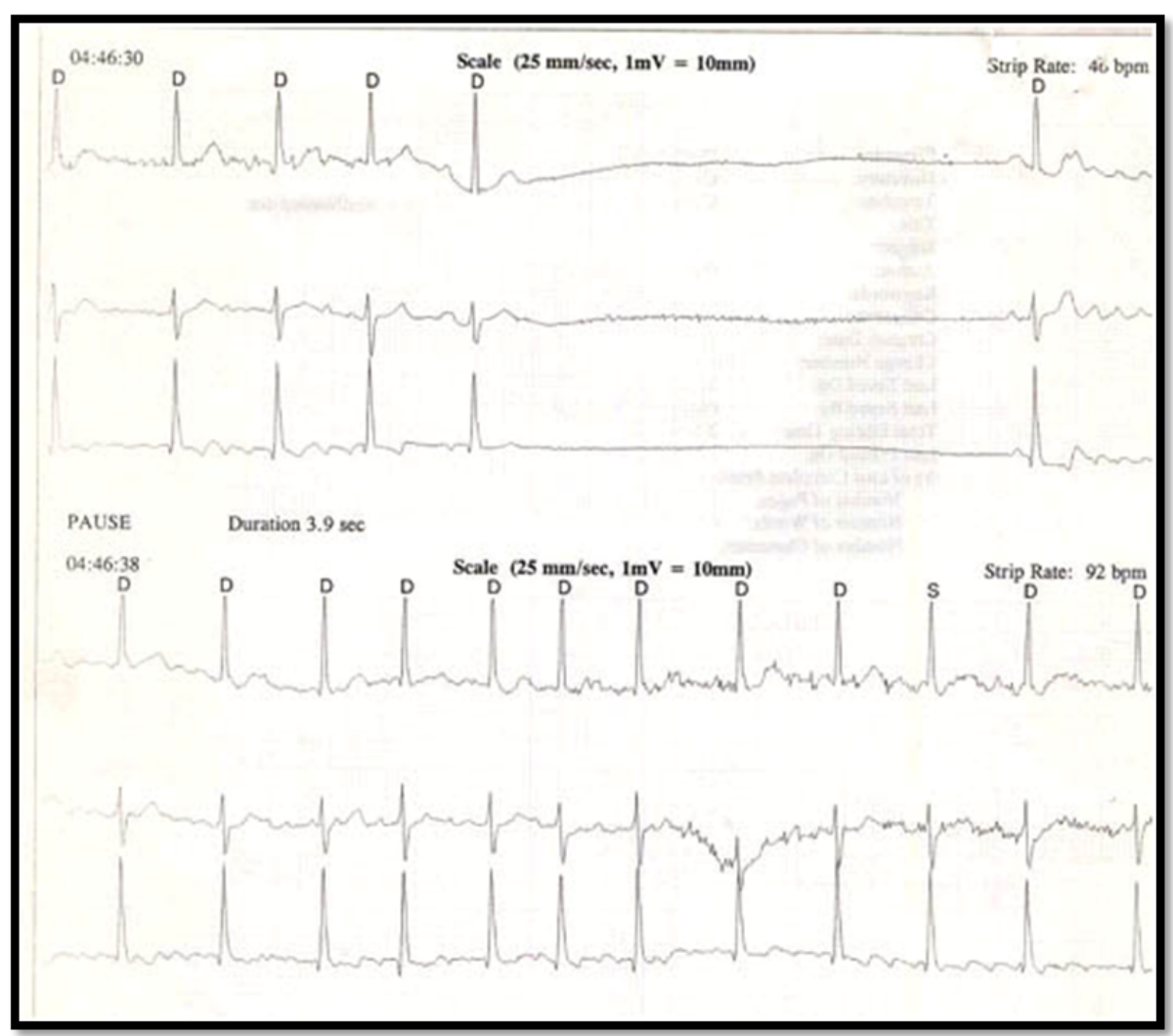

Figure 1 B: Holter monitor of the same patient in Figure 1 A. Showed symptomatic (SA) pause of 3.9 seconds followed by AF. 


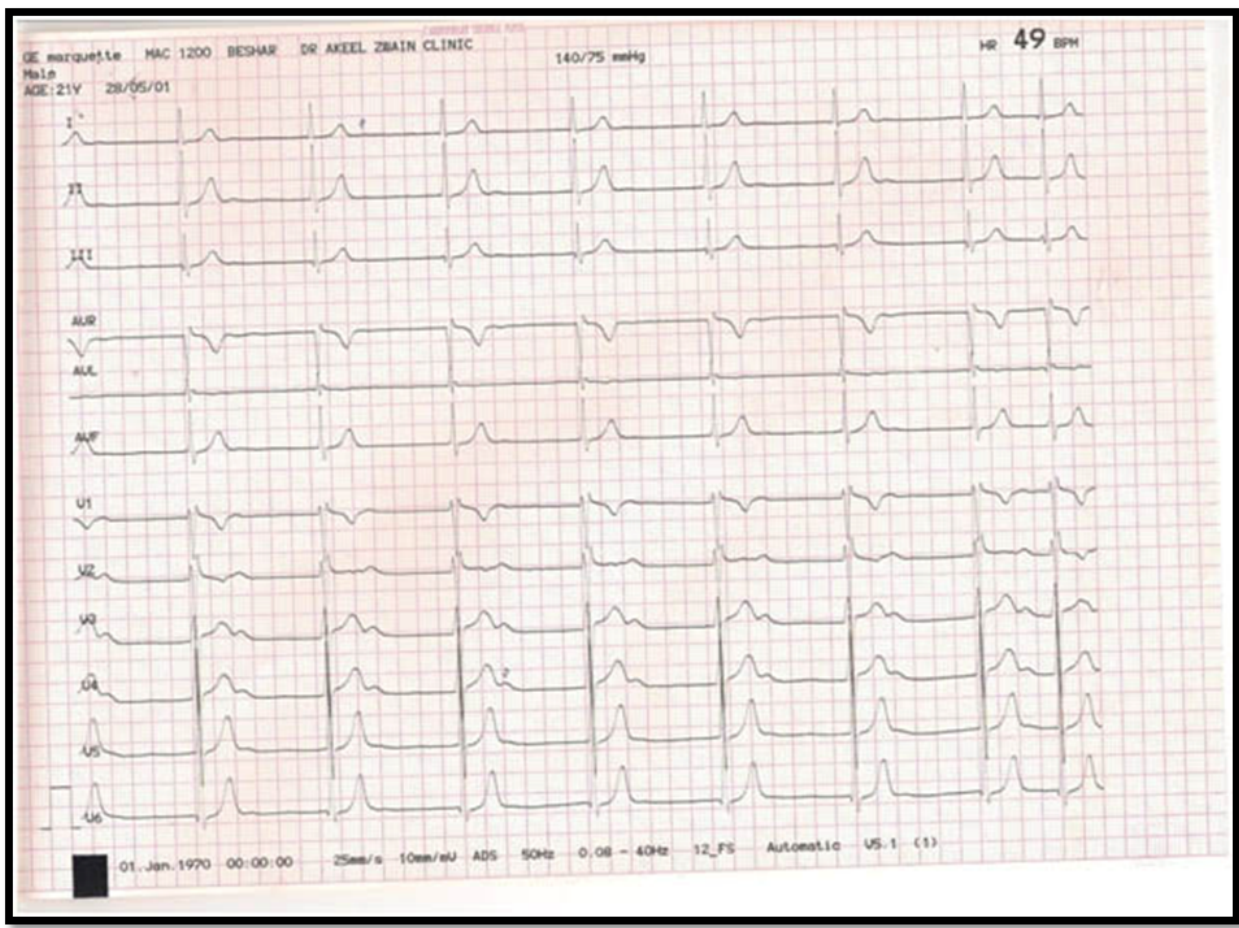

Figure 2 A: twenty-two years old young patient diagnosed as "hysterical" (neurogenic) syncope. ECG showed no sinus node P wave but junctional rhythm of 49bpm.

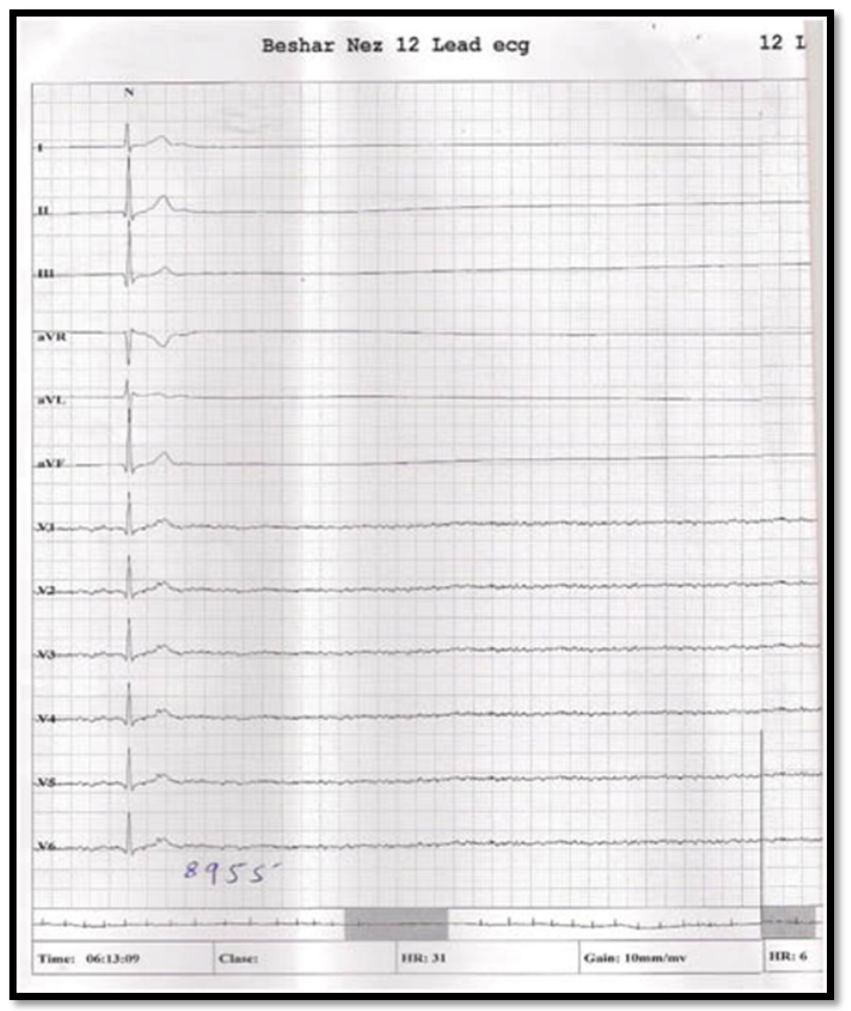

Figure 2 B: Holter record of the same patient in Figure $2 \mathrm{~A}$ documented severely symptomatic sinu-atrial (SA) pause of 9 seconds preceded by junctional escape beat. 


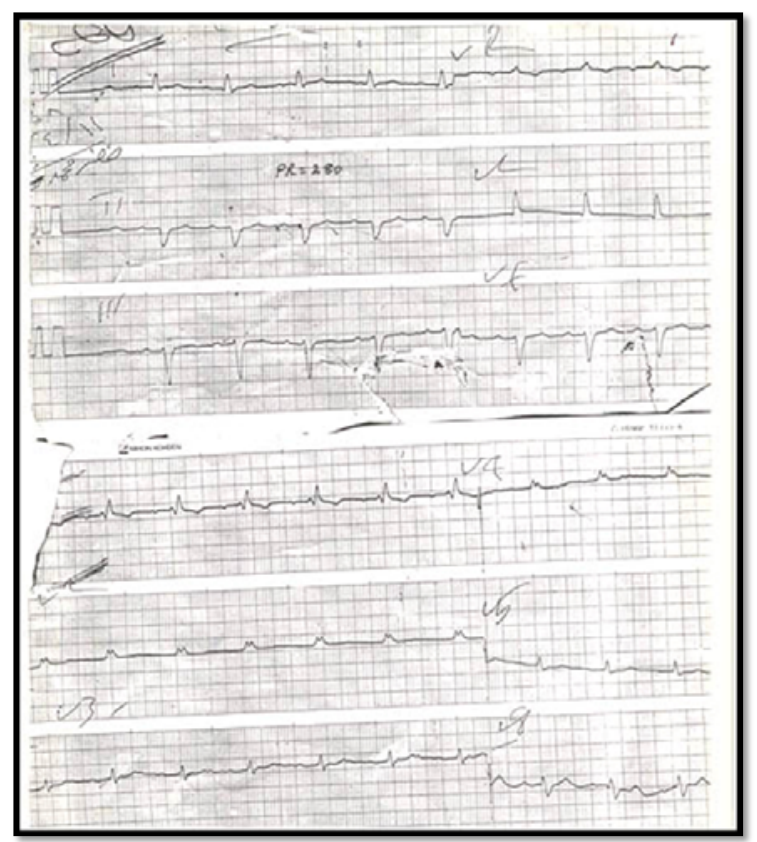

Figure 3 A: The 12 leads ECG of a $65 \mathrm{y}$ old man with recurrent syncope and dizzy spells, seen at neurology clinic and diagnosed and treated as vertebrobasilar insufficiency. ECG showed PR interval of 280msc, RBBB with left axis deviation (bifascicular block). ${ }^{22}$

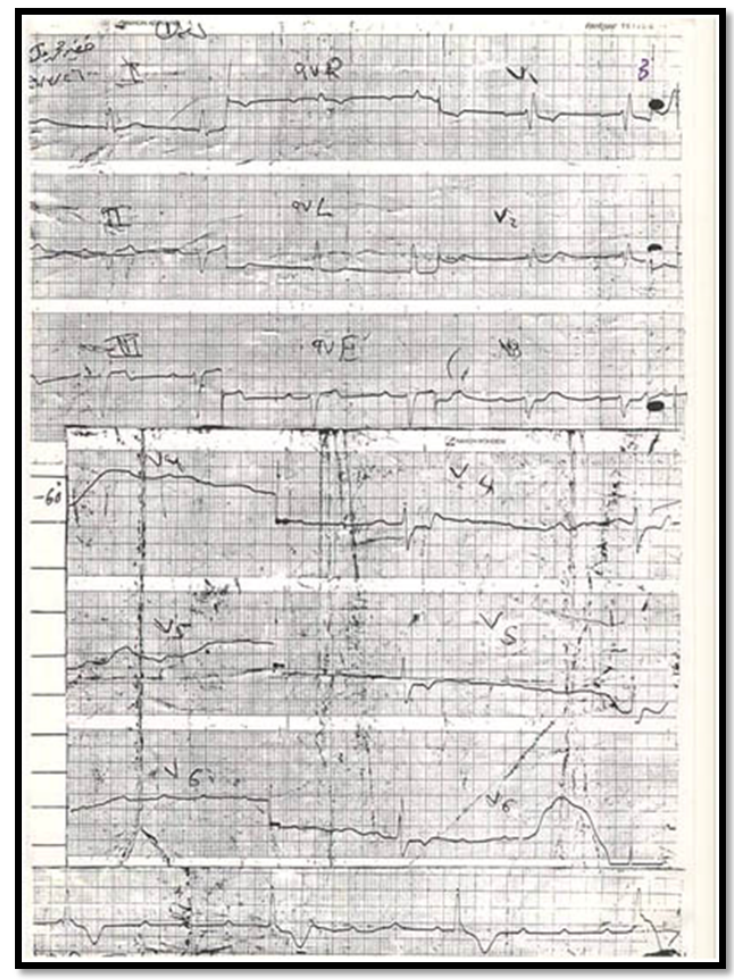

Figure 3 B: Technically very poor quality ECG of the same patient in Figure 3 A, showing bifascicular block degenerating into transient complete heart block at the bottom of the recording, the ECG was not carefully inspected. 




Figure 4 A: 60 y male patient presented with recurrent pre syncope and vague chest pain, gone through neurological investigations and coronary angiography (all normal). ECG showing LBBB long PR interval (bifascicular block).

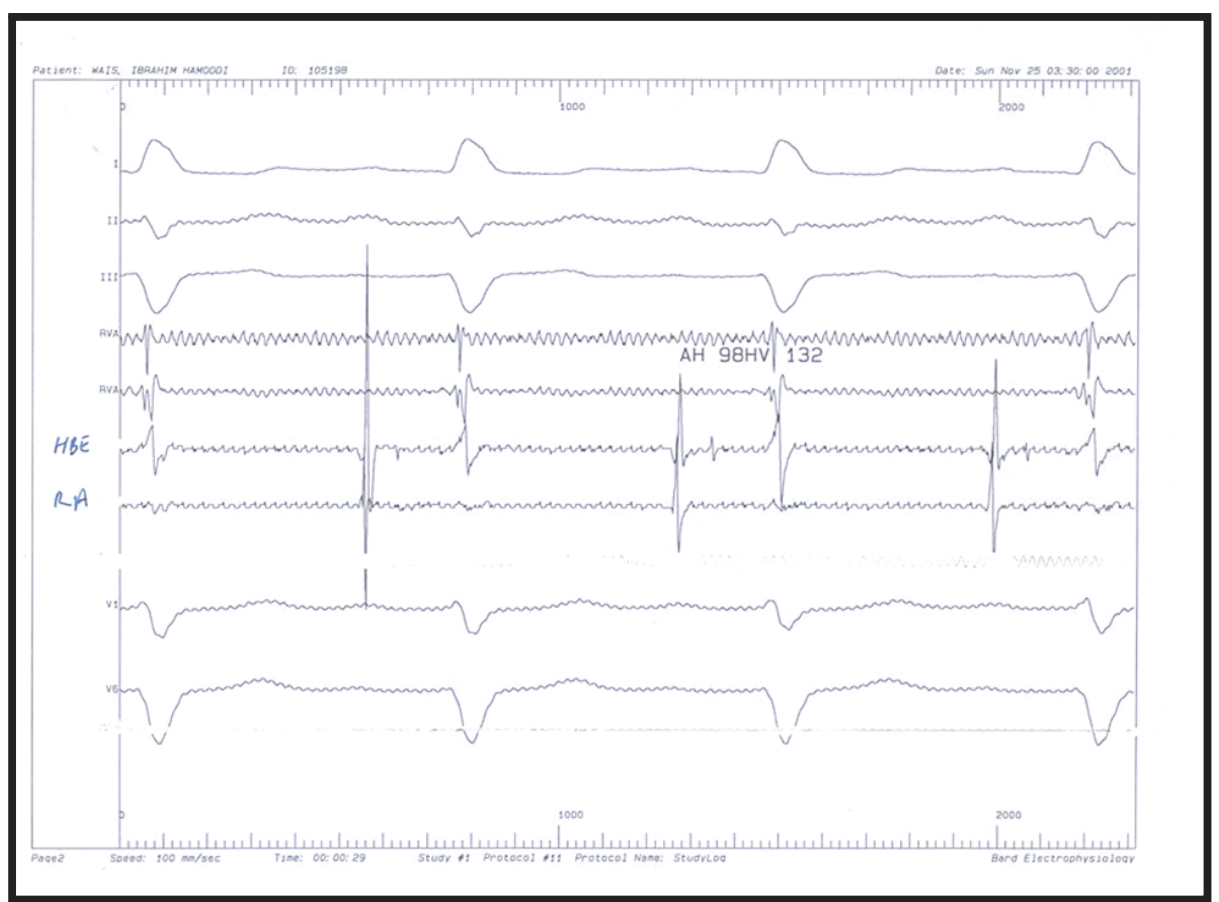

Figure 4 B: Electrophysiological study (EPS) of the patient in Figure $4 \mathrm{~A}$, at the His bundle channel (HBE) a prolonged below His interval (HV) of $132 \mathrm{msc}$ is seen.(Normal HV 50msc). Solid arrow $\mathrm{H}$, down arrow $\mathrm{V}^{22}$ 


\section{Discussion}

Missing the diagnosis of bradyarrhythmias in patients presenting with symptoms of syncope, pre syncope, or dizzy spells has a deleterious effect on the prognosis of this group of patients. ${ }^{8-17}$ The bradyarrhythmias diagnosis can be reached either by 12 leads ECG, Holter monitoring, or long term monitoring facilities including implantable loop recorder (ILR) or by EPS.,.$^{8,9,19-22}$ Figures 1-4 show samples of cases of misdiagnosed bradyarrhythmias. In a busy clinical practice, the rate of missing these cases of bradyarrhythmias is not rare..$^{7-10,19,20}$ Trying to understand and clarify the causes of this misdiagnosis is important to plan for management methodologies that can help minimize it to a great extent, which can be reflected on patient's survival. We found that the etiology of misdiagnosed was poor history taking (34\%), non-careful ECG inspection and interpretation (32\%), poor Holter recording (24\%), and non-considering EP studies (10\%). We found no similar studies dealing with the etiologies of misdiagnosed of bradyarrhythmias to compare our results with. The same is applied to the provisional diagnosis during the first or second medical contact before the bradyarrhythmias were diagnosed. We believe improving the above four etiologies of misdiagnosed bradyarrhythmias can be done through an organized continuing education program for concerned general physicians, clinical cardiologists, and general practitioners. These educational sessions should be case based, taken from real clinical life rather than textbook or lecture based. The other approach we adopted in this clinical survey is the direct feed back to the concerned medical practitioners who missed the diagnosis of bradyarrhythmias during the first, second, or third medical contact. Although this approach was not friendly welcomed by a few of the concerned practitioners, it was greatly appreciated by the majority of others. Some of them also attended the pacemaker implantation procedure for their patient and shared in the follow up. This is a single author case series study where the cases of misdiagnosed bradyarrhythmias were collected over a period of 10 years from four sources. Although the average per year number of misdiagnosed bradyarrhythmias cases looks small (15 cases per year) in this collective study, it may well indicate similar or more cases of bradyarrhythmias are missed in other practices per year.

\section{Conclusion}

Missing the diagnosis of bradyarrhythmias in patients presenting with symptoms of dizziness, pre syncope, and syncope is not rare in clinical practice. Good history taking and eye witness description, careful ECG inspection, proper Holter analysis, and considering EPS are major steps to help avoiding misdiagnose bradyarrhythmias. Educational program impact to minimize the misdiagnosis of bradyarrhythmias needs future study.

\section{Competing interests}

The author declares no competing interests.

\section{References}

1. Kusumoto FM, Schoenfeld $\mathrm{MH}$, Barrett $\mathrm{C}$, Edgerton JR, Ellenbogen KA, Gold MR, et al. 2018 ACC/AHA/HRS guideline on the evaluation and management of patients with bradycardia and cardiac conduction delay: A Report of the American College of Cardiology/American Heart Association Task Force on Clinical Practice Guidelines and the Heart Rhythm Society. Circul 2019; 140(8):e382-482.

2. Deal N. Evaluation of bradyarrhythmias in the emergency department. Emerg Med Pract 2013; 15(9):2-11.

3. BurriH, DayalN. Acute management of bradycardia in the emergency setting. Cardiovasc Med 2018; 21(04):98-104.

4. Weberndörfer V, Russi I, Kobza R. Bradyarrhythmias. Cardiovasc Med 2018; 21(09):218-26.

5. Sidhu S, Marine JE. Evaluating and managing bradycardia. Trends Cardiovasc Med 2019; 13:11.

6. Vogler J, Breithardt G, Eckardt L. Bradyarrhythmias and conduction blocks. Rev Esp Cardiol (English edition) 2012; 65(7):656-67. 
7. Chiesa V, Vignoli A, Paola Canevini M. Cardiac asystoles misdiagnosed as epileptic seizures. BMJ Case Rep 2015; 2015:bcr2014206969.

8. Breitenstein A. Long-term ECG recording system useful in patients with infrequent symptoms. Implantable loop recorders. Cardiovasc Med 2018; 21(03):66-8.

9. Bisignani A, De Bonis S, Mancuso L, Ceravolo G, Bisignani G. Implantable loop recorder in clinical practice. J Arrhythm 2018; 35(1):25-32.

10. Benditt DG. Syncope: an overview of diagnosis and treatment. Rev Urug Cardiol 2011; 26(1):3845.

11. Shen WK, Sheldon RS, Benditt DG, Cohen MI, Forman DE, Goldberger ZD, et al. 2017 ACC/ AHA/HRS Guideline for the Evaluation and Management of Patients with Syncope: A Report of the American College of Cardiology/American Heart Association Task Force on Clinical Practice Guidelines and the Heart Rhythm Society. Circul 2017; 136:e60-122.

12. Brignole M, Moya A, Lange FJ, Deharo JC, Elliott PM, Fanciulli A. 2018 ESC Guidelines for the diagnosis and management of syncope. Eur Heart J 2018; 39, 21(1):1883-948.

13. Patel PR, Quinn JV. Syncope: a review of emergency department management and disposition. Clin Exp Emerg Med 2015; 2(2):6774.

14. Krahn A, Klein G. Syncope. Circ 2013; 127:1330 -9 .

15. Grossman SA, Badireddy M. Syncope. Emerg Med J 2019; 36(2):108-16.

16. Lisboa Da Silva RMF. Syncope: Epidemiology, etiology, and prognosis. Front Physiol 2014; 5:471.

17. Runser LA, Gauer RL, Houser A. Syncope: Evaluation and differential diagnosis. Am Fam Physician 2017; 95(5):303-12.

18. Albassam OT, Redelmeier RJ, Shadowitz S, Husain AM, Simel D, Etchells EE. Did this patient have cardiac syncope? The rational clinical examination systematic review. JAMA 2019; 321(24):2448.

19. O'Brien H, Anne Kenny R. Syncope in the elderly. ECR 2014; 9(1):28-36.

20. Khan T, Stecker MA, Stecker M. Evaluating the patient with loss of consciousness. Surg Neuro Int 2015; 6(Suppl 6):S262-5.

21. Semelka M, Gera J, Usman S. Sick sinus syndrome: A review. Am Fam Physician 2013; 87(10):691-6.

22. Martin-Almor J, Cladellas M, Bazan V, Delclos J. Novel predictors of progression of atrioventricular block in patients with chronic bifascicular block. Rev Espa de Cardiol 2010; 63(4):400-8. 